\title{
Basic Hypothesis and Therapeutics Targets of Depression: A Review
}

\author{
Monika Kadian, Hemprabha Tainguriya, Nitin Rawat, Varnika Chib, Jeslin Johnson and Anil Kumar* \\ Pharmacology Division, University Institute of Pharmaceutical Sciences, UGC Centre of Advanced Study, Panjab University, Chandigarh 160014, India
}

*Corresponding author: Dr. Anil Kumar, PhD, Professor of Pharmacology, Pharmacology division, University Institute of Pharmaceutical Sciences, Panjab University, Chandigarh 160014, India.

Received Date: May 11, 2021

Published Date: June 07, 2021

\begin{abstract}
Depression is a psychological disorder marked by emotional symptoms such as melancholy, anhedonia, distress mood, loss of interest in daily life activities, feeling of worthlessness, sleep disturbances and destructive tendencies. According to WHO, more than 264 million people from all age groups are suffering with depression thus, it is become a leading cause of disability and infirmity worldwide. It is estimated that $40 \%$ of risk for depression is genetic and the other $60 \%$ is non-genetic which involved acute \& chronic stress, childhood trauma, viral infections and even random processes during brain development. Depression is mainly due to neurotransmitter imbalances, HPA disturbances, increased oxidative and nitrosative damage, impairment in glucose metabolism, and mitochondrial dysfunction, etc. The monoamine hypothesis is based on attenuation of monoamines such as serotonin (5-HT), norepinephrine (NE) and dopamine (DA) in the brain regions (hippocampus, limbic system and frontal cortex) that can cause depression like symptoms. Depression is also marked by increased level of corticotrophin-releasing hormone (CRH) and and impaired responsiveness to glucocorticoid hormone. The present review emits about its background knowledge, various pathophysiological pathways involved and current therapy for the depression.
\end{abstract}

Keywords: Depression, HPA axis, mitochondrial dysfunction, NMDA receptor, Serotonin, Glutamate

\section{Introduction}

Depression is one in all the two diagnostic classes that represent common mental disorders (CMDs), the other is anxiety disorder. Both are highly widespread among the community and influence the mood or feelings of affected persons. Depressive disorders are characterized by dejection, loss of pleasure, guilt feelings or low selfworth, insomnia, appetite imbalance, feelings of tiredness, and loss of concentration. At a worldwide level, according to WHO 2020 more than 264 million people from all age group are suffering with depression, equivalent to $4.4 \%$ of the world's population. India is home to an estimated fifty seven million people $18 \%$ of the the world estimate) littered with depression [1]. Depression may be caused due to neurotransmitter imbalances [2], increased oxidative and nitrosative damage in brain, HPA disturbances, impairment in glucose metabolism, and mitochondrial dysfunction, etc [3]. The monoamine hypothesis is based on attenuation of monoamines such as serotonin (5-HT), norepinephrine (NE) and dopamine (DA) in the brain regions (hippocampus, limbic system and frontal cortex) that can cause depression like symptoms. Monoaminergic neurotransmitters are metabolised by monoamine oxidase (MAO) [4,5]. Decreased antioxidant status, elevated oxidative and nitrosative stress are found in patients with depression [6]. Moreover, there is greater secretion of corticotrophin-releasing hormone (CRH) and impaired responsiveness to glucocorticoids [7]. The hypothalamic-pituitary-adernal (HPA) axis hyperactivity is present in around $50 \%$ of depressed patients (80\% if severely depressed). 
Decreased antioxidant concentration, and raised oxidative and nitrosative stress are major marker that are present in patients with major depression [8]. This is evidenced by reduced level of antioxidants such as vitamin C [9] vitamin E $[10,11]$ and coenzyme Q10 [12] in plasma and by reduced antioxidant enzyme activity such as glutathione peroxidase [13]. These paucities in antioxidant defence system impaired the protection against reactive oxygen species (ROS), which lead to damaged fatty acids, proteins and DNA. In depression, there is rise in the lipid peroxidation levels, encompassing increase in malondialdehyde [14-16] and oxidative damage to DNA, characterized by increased levels of 8-hydroxy-2deoxyguanosine [17]. Furthermore, elevated oxidative stress level in brain is reflective of neurotoxicity and neuronal death towards the progression of chronic stress-induced depressive disorders [18]. Nitric oxide (NO) is an important modulator of depression $[19,20]$ because NO production is increased in depression. Moreover, In brain, No is an essential neurotransmitter that normalize depression, behavioral, cognitive and emotional processes [21]. In experimental animals, plasma nitrate levels have been increased significantly under stressful condition [22]. Psycho-social and physical stressors can inhibit the neurogenesis in various animal models and thus lead to decreased cell proliferation and survival [23]. The types of depression are depicted in Figure 1.

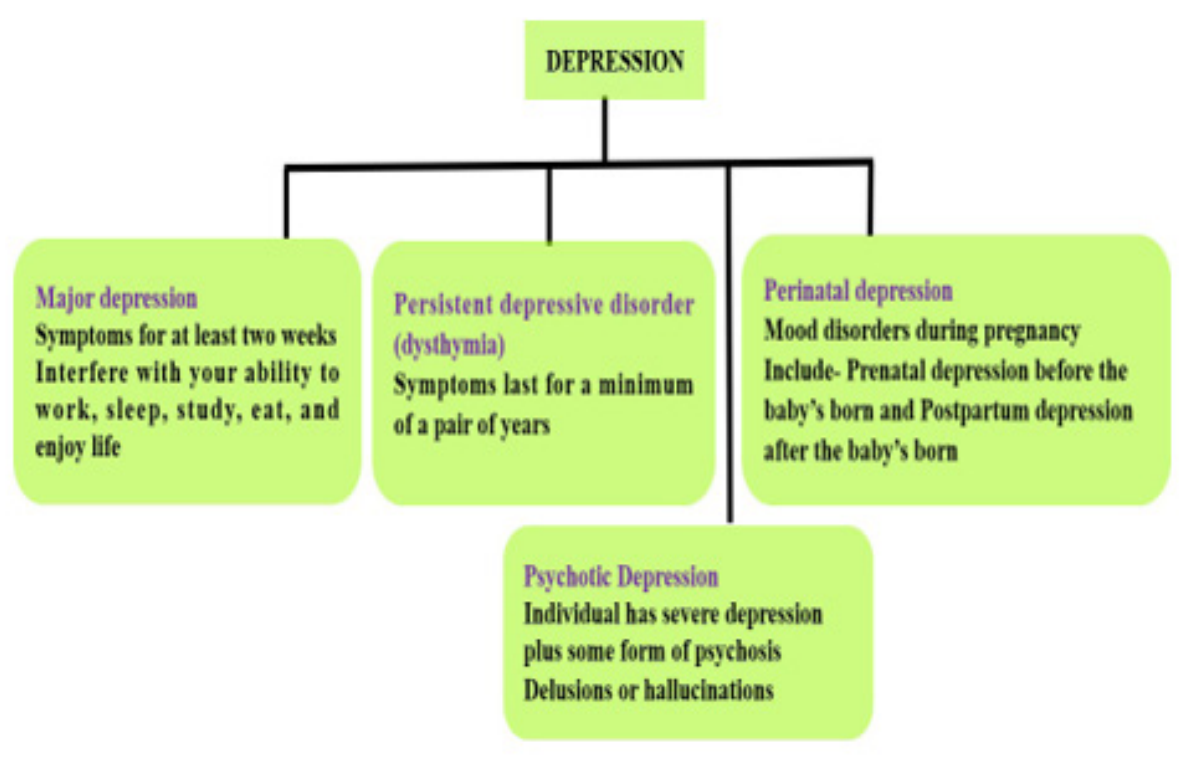

Figure 1: Types of depression.

\section{Pathophysiological Hypotheses of Depression}

Following neurotransmitters and receptor systems have been reported to be involved in pathophysiology of depression (Figure 2\&3)

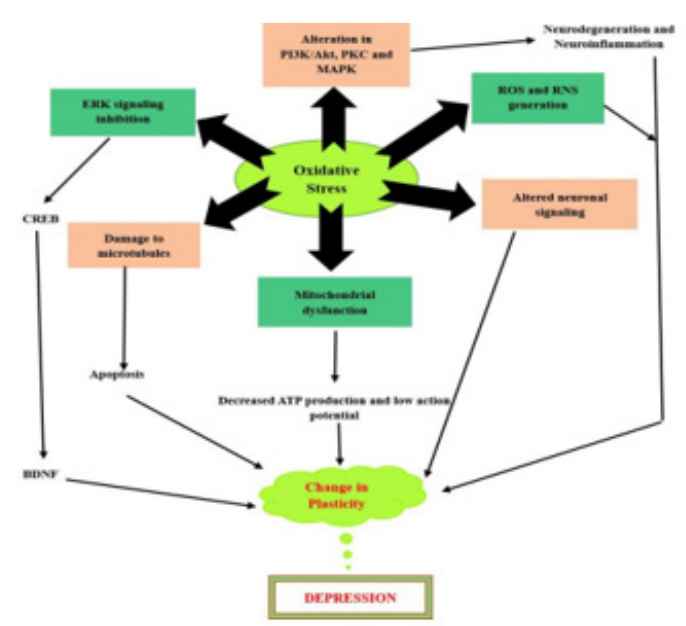

Figure 2: The different pathological mechanisms of depression. 


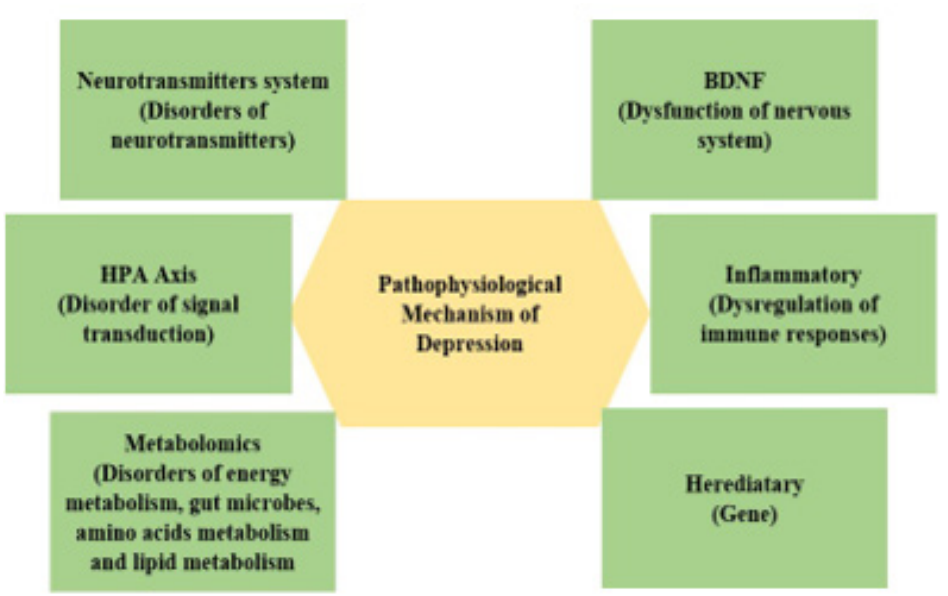

Figure 3: The different pathological mechanisms of depression.

\section{Role of monoaminergic system/ monoamine theory}

Basic hypothesis of depression firstly described about the functional inadequacy of adrenergic neurotransmitters such as noradrenaline (NAdr), serotonin (5-HT) and dopamine (DA). Facts for this hypothesis obtained from the animal experiments and clinical studies, which depicted that the reserpine (an antihypertensive drug), a monoamine antagonist lead to the reduction of pre-synaptic stores of NAdr, DA and 5-HT, was used to treat things like high blood pressure, is rarely used at the present time due to the fact that depression is a common side effect. Thus, not solely monoamine agonists decrease depression, however monoamine antagonists (Reserpine) will induce depression [24]. The enzyme (monoamine oxidase) which plays an essential role in the malfunctioning and oxidation of monoamines like DA, NE and 5-HT. The monoamine oxidase A showing targeted preferred action on serotonin, and its an important target for screening antidepressant activity of monoamine oxidase inhibitors [24].

\section{Neuronal framework of depression}

Several reported literature provides the evidences of deformities in various parts of the brain that are the main driven force for controlling emotion, functions and reward outcomes. Postmortem and neuroimaging examination have depicted the changes in morphology which is illustrated by reduced grey-matter volume and interstitial tissue density in the hippocampus and anterior cortex, which is the main focused region in animal analysis of depression. The decline in hippocampal operate, that is believed to possess a repressive result on the hypothalamic-pituitary- adrenal (HPA) axis, might probably be answerable for the hypercortisolemia seen in depression [25].

\section{Serotonin hypothesis}

Serotonin(5-hydroxy tryptamine), a monoamine with a large distribution throughout the CNS. It is concerned in physical activities like pain sensation, appetence regulation, aggression and mood. This terminology mainly based on the deficiency in 5-HT as the foremost cause of depression, which can be altered by antidepressants, which would bring back the actual functioning in the affected subjects. As approved by the post-mortem examination, neurochemical, genetic neuroimaging and pharmacological studies that a purposeful insufficiency in 5-HT neuronal firing within brain regions which is known to play role in controlling emotions have systematically been related to facet of the MDD pathophysiology. Moreover, subsequently SSRIs solely were seem to be efficient to treat the symptoms of depression. This fact more reinforced the involvement of 5-HT within the pathological process of the illness [26]. 5-HT is implicated in MDD, and the genesis of this disease may be linked to the dysfunctioning of 5-HT receptors [27]. It has been reported that 5-HT sensitivity (reduction of 5-HT/tryptophan), is more common in MDD patients [28]. Membrane transporters which help in translocation of 5-HT across the membrane are basically proteinaceous in nature and mostly targeted for the treatment of depression. These proteins help in the regulation of serotonergic transmission that are also associated with MDD.

\section{Role of hypothalamic-pituitary-adrenal (HPA) axis sys- tem}

The response systems for stress are mainly regulated by the HPA-axis. In the central nervous system when HPA-axis starts stimulation, than an individual's recognize the fearful and noxious stimulus (e.g., a traumatic episodes) from the surrounding [29]. Various researchers noted that the HPA-axis showing sensitivity towards psychological ans social stress agents, which is observed by alteration in cortisol levels which is accompanied with the increased risk of producing altered psychological symptoms in the affected population [29]. HPA- axis is activated in response to the traumatic episodes by a signal within the para-ventricular nucleus (PVN) of the hypothalamus. Neurons in the PVN synthesize and secrete corticotrophin-releasing hormone (CRH), which binds to specific receptors in the anterior pituitary. Then the anterior 
pituitary specifically adrenal cortex stimulates the production and release of adrenocorticotropin hormone (ACTH) [30]. High cortisol or corticosterone levels may be the key factor in precipitating the symptoms of depression by impairing brain functions like neurogenesis, neuronal survival, neuronal excitability and plasticity (Figure 4) [31].

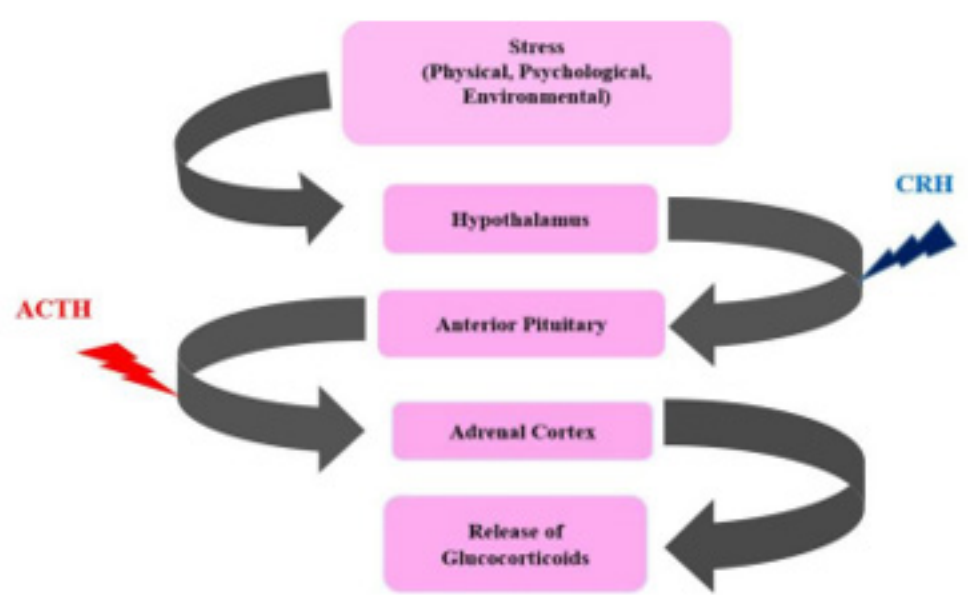

Figure 4: Stress and HPA axis. CRH: Corticotrophin-releasing hormone, ACTH: Adrenocorticotropin hormone

\section{Role of neurotrophins}

The most common and extensively dispersed neurotrophin is the brain-derived neurotrophic factors (BDNF) within the central nervous system. BDNF is liable for controlling cognitive functions, synaptic plasticity and neurogenesis, which is directly linked to the pathogenesis of depression [32,33]. There is decrease of brain BDNF levels in depression patients [34]. The raised BDNF levels improves depressive behaviors so that it is the area of interest for researchers for the antidepressant therapy [35].

\section{Role of cytokines}

Pro-inflammatory cytokines like interleukin (IL)-1 $\beta$, IL-6, tumor necrosis factor á (TNF- $\alpha$ ) and interferon- $\gamma$ play an important role in pathophysiology of depression. Up-regulation of these proinflammatory cytokines were identified in depressed patients. Antidepressants decreased the levels of pro-inflammatory cytokines and improved symptoms of depression symptoms [36,37].

\section{Role of Nrf-2 in depressive disorders}

Nuclear factor (erythroid-derivative 2) is a transcription factor that plays a central role in cellular defense against oxidation and electrophilic insults. Nrf2, situated in the gene's promoter domain adhere to the antioxidant response elements (ARE) encodes several antioxidant and enzymes responsible for detoxification and stressrelated responsive proteins. Interruption or failure of Nrf2 signaling pathway lead to the improved susceptibility towards the oxidative and electrophilic stresses and also injuries in the inflammatory tissues. Since Nrf2 has been recently described to play a crucial role in regulating inflammation, and inflammation has been related to depression. Furthermore, a relationship between Nrf2 and protective effects have also been described in the central nervous system (CNS) [38]. Nrf2 stimulates Heme oxygenase-1 (HMOX1, HO-1) and this enzyme catalyzes the breakdown of heme into biliverdin, carbon monoxide, and iron which is the beneficial aid for antidepressant action $[39,40]$.

\section{Involvement of Extracellular signal-regulated kinase (ERK) in depression}

Table 1: Current therapeutic targets for depression [52].

\begin{tabular}{|l|c|c|}
\hline \multicolumn{2}{|c|}{ Therapeutic targets } & Description \\
\hline & Infliximab & Tnflammatory pathway hypothesis: \\
\hline & Sirukumab & IL-6 Antagonists \\
\hline & Acetylsalicylic acid & Irreversibly inhibits cyclooxgenase-1 and -2 \\
\hline & Celecoxib & COX-2 inhibitor \\
\hline & Omega-3 polyunsaturated fatty acids & Natural anti-inflammatory agents \\
\hline & Curcumin & Natural anti-inflammatory agents \\
\hline & Doxycycline & Inflammatory mouse model, doxycycline reduces the incidence and severity of \\
& &
\end{tabular}




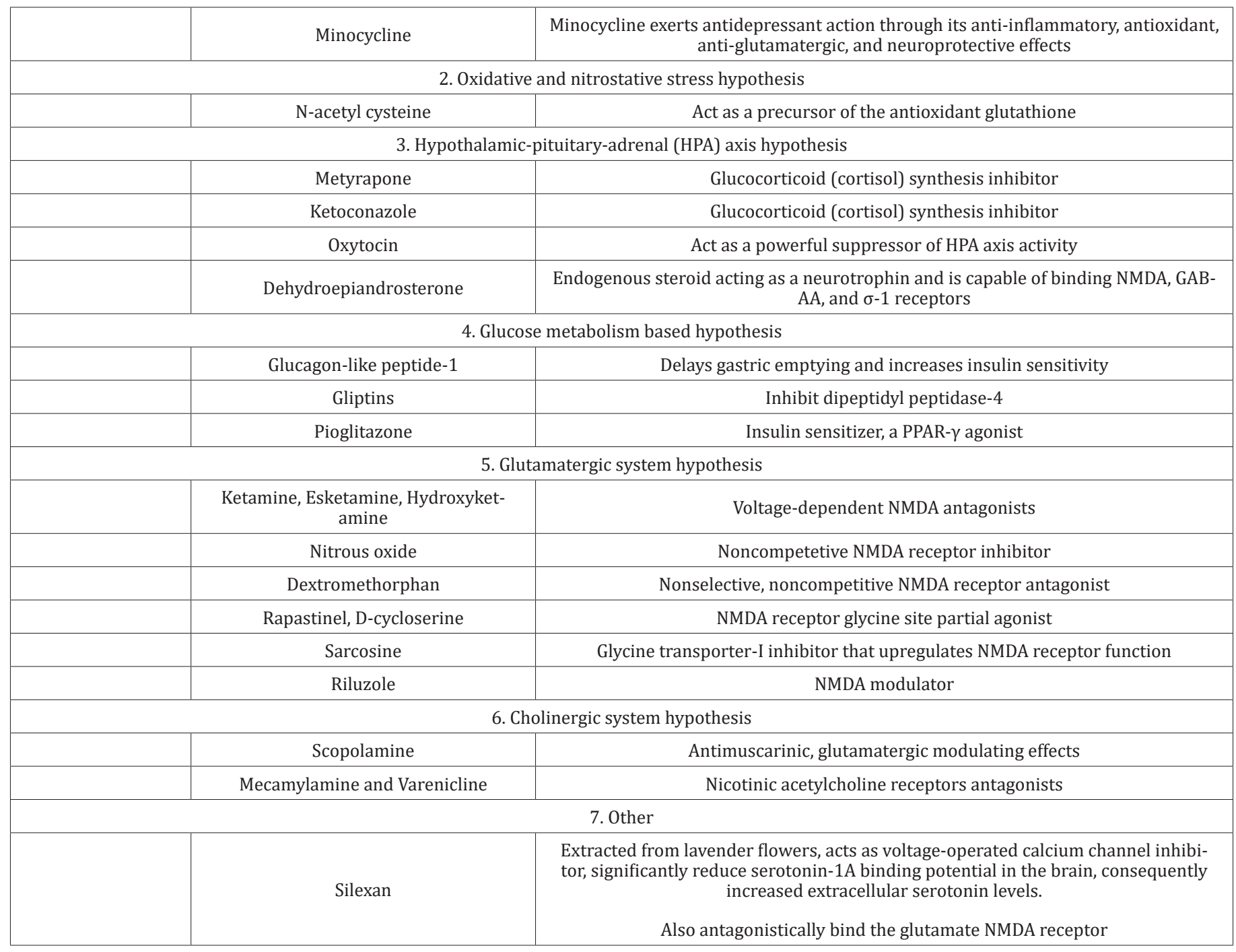

COX: Cyclooxygenase; IL-6: Interleukin-6; GABA-A: Gamma amino-butyric acid A; NMDA: N-methyl-D-aspartate; PPAR-ץ: Peroxisome proliferator-activated receptor gamma; TNF- $\alpha$ : Tumor necrosis factor-alpha.

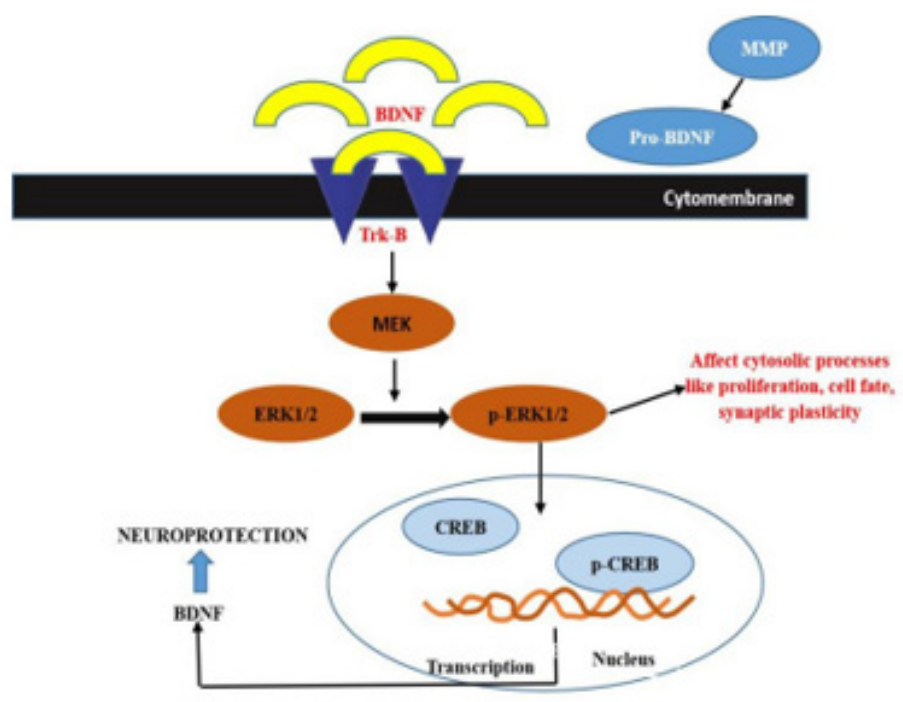

Figure 5: The ERK Signalling pathway involved in depression. 
Mitogen-activated protein kinases (MAPKs) family consisting extracellular signal-regulated kinases (ERK1 and ERK2) [41], a downregulated target of BDNF, is stimulated by the binding of BDNF to tyrosine kinase receptor-B (Trk-B) in the Ras-dependent pathway. Moreover, this eventually leads to the phosphorylation of cAMP response element-binding protein (CREB) [42,43]. Various experimental studies have evidenced that the diminshed expression of ERK1/2 results in depressive behaviour in rodents $[44,45]$. Decreased ERK expression and activation have been found in the brain of suicidal persons with depression in autopsy [46]. Antidepressants can alleviate the altered effects of depression by the escalation in ERK1/2 [47] and p-ERK1/2 expression (Figure 5) [48-52].

\section{Conclusion}

In the present review we have described about the pathophysiological targets and targeted therapeutic for depression. There is an immense demand for a novel effective and efficacious therapeutic agents and therapeutic targets as the disease becoming very common in our all age groups. At present so many marketed formulation available but only few consumers getting a clinically significant benefits. Therefore we need to explore all the hypothesis deeply and try to understand the underlying mechanism in more possible ways.

\section{Acknowledgement}

We gratefully acknowledge the support provided by the Indian Council of Medical Research (ICMR), New Delhi, India, ICMR-SRF grant (No.45/35/2018-PHA/BMS).

\section{Conflict of Interest}

Authors declare no conflict of interest.

\section{References}

1. Thompson Scott M, Kallarackal Angy J, Kvarta Mark D, Adam M Dyke Van, LeGates Tara A, et al. (2015) An excitatory synapse hypothesis of depression. Trends Neurosci 38(5): 279-294.

2. Hou Zhenghua, Jiang Wenhao, Yin Yingying, Zhang Zhijun, Yuan Yonggui (2016) The Current Situation on Major Depressive Disorder in China: Research on Mechanisms and Clinical Practice. Neurosci Bull 32(4): 389397.

3. Rothkirch Marcus, Tonn Jonas, Kohler Stephan, Sterzer Philipp (2017) Neural mechanisms of reinforcement learning in unmedicated patients with major depressive disorder. Brain 140(4): 1147-1157.

4. Delgado P, Moreno F (1999) Antidepressants and the brain. Int Clin Psychopharmacol 14 (1): S9-S16.

5. Tanabe A, Nomura S, Nihon Rinsho (2007) Pathophysiology of depression. Nihon Rinsho 65(9): 1585-1590.

6. Zhang Xianhua, Han Linran, Liu Jin, Xu Qiuyue, Guo Yuxin, et al. (2018) Pharmacokinetic Study of 7 Compounds Following Oral Administration of Fructus Aurantii to Depressive Rats. Front Pharmacol 9: 131.

7. Pariante CM, Lightman SL (2008) The HPA axis in major depression: classical theories and new developments. Trend Neurosci 31: 464-468.

8. Maes M, Mihaylova I, Kubera M, Uytterhoeven M, Vrydags N, et al. (2011) Lower whole blood glutathione peroxidase (GPX) activity in depression, but not in myalgic encephalomyelitis/chronic fatigue syndrome: another pathway that may be associated with coronary artery disease and neuroprogression in depression. Neuroendocrinol Lett 32: 133-140.
9. Khanzode SD, Dakhale GN, Khanzode SS, Saoji A, Palasodkar R (2003) Oxidative damage and major depression: the potential antioxidant action of selective serotonin re-uptake inhibitors. Redox Rep 8: 365-370.

10. Maes M, De Vos N, Pioli R, Demedts P, Wauters A, et al. (2000) Lower serum vitamin $\mathrm{E}$ concentrations in major depression. Another marker of lowered antioxidant defenses in that illness. J Affect Disord 58: 241-246.

11. Owen AJ, Batterham MJ, Probst YC, Grenyer BF, Tapsell LC (2005) Low plasma vitamin E levels in major depression: diet or disease? Eur J Clin Nutr 59: 304-306.

12. Maes M, Mihaylova I, Kubera M, Uytterhoeven M, Vrydags N, et al. (2009) Increased 8-hydroxy-deoxyguanosine, a marker of oxidative damage to DNA, in major depression and myalgic encephalomyelitis/chronic fatigue syndrome. Neuroendocrinol Lett 30: 715-722.

13. Maes M, Galecki P, Chang YS, Berk M (2011) A review on the oxidative and nitrosative stress (O \& NS) pathways in major depression and their possible contribution to the (neuro) degenerative process in that illness. Prog NeuroPsychopharmacol Biol Psychiatry 35: 676-692.

14.Ozcan ME, Gulec M, Ozerol E, Polat R, Akyol O (2004) Antioxidant enzyme activities and oxidative stress in affective disorders. Int. Clin. Psychopharmacol 19: 89-95.

15. Sarandol A, Sarandol E, Eker SS, Erdinc S, Vatansever E, et al. (2007) Major depressive disorder is accompanied with oxidative stress: short term antidepressant treatment does not alter oxidative-antioxidative systems. Hum Psychopharmacol 22: 67-73.

16. Wei YC, Zhou FL, He DL, Bai JR, Hui LY, et al. (2009) The level of oxidative stress and the expression of genes involved in DNA-damage signaling pathways in depressive patients with colorectal carcinoma. J Psychosom Res 66: 259-266.

17. Maes M, Mihaylova I, Kubera M, Uytterhoeven M, Vrydags N, et al. (2009) Lower plasma coenzyme Q10 in depression: a marker for treatment resistance and chronic fatigue in depression and a risk factor to cardiovascular disorder in that illness. Neuroendocrinol Lett 30: 462469.

18. Tsuboi H, Tatsumi A, Yamamoto K, Kobayashi F, Shimoi K, et al. (2006) Possible connections among job stress, depressive symptoms, lipid modulation and antioxidants. J Affect Disord 91: 63-70.

19. Yildiz F, Erden BF, Utkan GUT, Gacar N (2000) Antidepressantlike effect of 7- nitroindazole in the forced swimming test in rats. Psychopharmacology 149: 41-44.

20. Wang W, Luo X, Li H (2010) Terahertz and Infrared Spectra of Plumbagin, Juglone, and Menadione. Carniv. Plant Newsl 39(3): 82-88.

21. Baranano ED, Ferris CD, Snyder SH (2001) Atypical neural messengers. Trends Neurosci 24(2): 99-106.

22. Lee CY, Cheng HM, Sim SM (2007) Mulberry leaves protect rat tissues from immobilization stress-induced inflammation. Biofactors 31(1): 25-33.

23. Joels M, Karst H, Krugers HJ, Lucassen PJ (2007) Chronic stress: implications for neuronal morphology, function and neurogenesis. Front Neuroendocrinol 28(2-3): 72-96.

24. Sacher J, Houle S, Parkes J, Rusjan P, Sagrati S, et al. (2011) Monoamine oxidase A inhibitor occupancy during treatment of major depressive episodes with moclobemide or St. John's wort: an [11C]-harmine PET study. J Psychiatry Neurosci 36(6): 375-382.

25. Maes Michael, Galecki Piotr, Chang Yong Seun, Berk Michael (2011) A review on the oxidative and nitrosative stress (O\&NS) pathways in major depression and their possible contribution to the (neuro)degenerative processes in that illness. Prog. Neuro-Psychopharmacol Biol Psychiatry 35(3): 676-692

26. Millan Mark J (2004) The role of monoamines in the actions of established and "novel" antidepressant agents: a critical review. Eur J Pharmacol 500(1-3): 371-384. 
27. Fakhoury Marc (2016) Revisiting the serotonin hypothesis: implications for major depressive disorders. Mol Neurobiol 53(5): 2778-2786.

28. Albert Paul R, Chawki Benkelfat, Laurent Descarries (2012) The neurobiology of depression-revisiting the serotonin hypothesis. Cellular and molecular mechanisms. Philos Trans R Soc Lond B Bio Sci 367(1601): 2378-2381.

29. Gunnar Megan, Quevedo Karina (2007) The Neurobiology of Stress and Development. Annu Rev Psychol 58: 145-173.

30. Finberg John P.M, Rabey Jose M (2016) Inhibitors of MAO-A and MAO-B in Psychiatry and Neurology. Front Pharmacol 7: 340.

31. Krishnan Vaishnav, Nestler Eric J (2008) The molecular neurobiology of depression. Nature 455(7215): 894-902.

32. Cowen Philip J (2008) Serotonin and depression: pathophysiological mechanism or marketing myth? Trends Pharmacol Sci 29(9): 433-436.

33. Saldanha Brig D, Kumar Maj N, Ryali Surg Capt VSSR, Srivastava K, Pawar Surg Capt AA (2009) Serum Serotonin Abnormality in Depression. Med J Armed Forces India 65(2): 108-112.

34. Papadimitriou Anastasios, Priftis Kostas N (2009) Regulation of the Hypothalamic-Pituitary-Adrenal Axis. Neuroimmunomodulation 16(5): 265-271.

35. Sousa Nuno, Cerqueira João J, Almeida Osborne FX (2008) Corticosteroid receptors and neuroplasticity. Brain Res Rev 57(2): 561-570.

36. Michel Tanja Maria, Pülschen Dietrich, Thome Johannes (2012) The Role of Oxidative Stress in Depressive Disorders. Curr Pharm Des 18(36) 5890-5899.

37. Black Catherine N, Bot Mariska, Scheffer Peter G, Cuijpers Pim, Penninx Brenda WJH (2015) Is depression associated with increased oxidative stress? A systematic review and meta-analysis. Psychoneuroendocrinology 51: 164-175.

38. Valko Marian, Leibfritz Dieter, Moncol Jan, Cronin Mark TD, Mazur Milan, et al. (2007) Free radicals and antioxidants in normal physiological functions and human disease. The Int J Biochem Cell Biol 39(1): 44-84.

39. Grases G, Colom M A, Fernandez R A, Costa-Bauzá A, Grases F (2014) Evidence of Higher Oxidative Status in Depression and Anxiety. Oxid Med Cellular Longev 2014(12): 1-5.

40. Michel Tanja Maria, Frangou Sophia, Thiemeyer Dorothea, Camara Sibylle, Jecel Julia, et al. (2007) Evidence for oxidative stress in the fronta cortex in patients with recurrent depressive disorder - a postmortem study. Psychiatry Res 151(1-2): 145-150.

41. Vaváková Magdaléna, uraIková Zdenka, Trebatická Jana (2015) Markers of Oxidative Stress and Neuroprogression in Depression Disorder. Oxid Med Cell Longev 2015(6): 1-12.

42. Fridovich Irwin (1986) Biological Effects of the Superoxide Radical. Arch Biochem Biophys 247(1): 1-11.

43. Block Gladys, Dietrich Marion, Norkus Edward P, Morrow Jason D, Hudes Mark, et al. (2002) Factors Associated with Oxidative Stress in Human Populations. Am J Epidemiol 156(3): 274-285.

44. Aschbacher Kirstin, O’Donovan Aoife, Wolkowitz Owen M, Dhabhar Firdaus S, Su Yali, et al. (2013) Good stress, bad stress and oxidative stress: Insights from anticipatory cortisol reactivity. Psychoneuroendocrinology 38(9): 1698-708.

45. Araujo Jesus A (2011) Particulate air pollution, systemic oxidative stress, inflammation, and atherosclerosis. Air Qual Atmos Health 4(1): 79-93.

46. Isik Birgul, Ceylan Ali, Isik Recep (2007) Oxidative Stress in Smokers and Non-smokers. Inhal Toxicol 19(9): 767-769.

47. Doi Kunio, Uetsuka Koji (2011) Mechanisms of Mycotoxin-Induced Neurotoxicity through Oxidative Stress-Associated Pathways. Int J Mol Sci 12(8): 5213-5237.

48. Faraut Brice, Boudjeltia Karim Zouaoui, Vanhamme Luc, Kerkhofs Myriam (2012) Immune, inflammatory and cardiovascular consequences of sleep restriction and recovery. Sleep Med Rev 16(2): 137-149.

49. Martinowich Keri, Lu Bai (2008) Interaction between BDNF and Serotonin: Role in Mood Disorders. Neuropsychopharmacol 33(1): 73 83.

50. Martinowich Keri, Manji Husseini, Lu Bai (2007) New insights into BDNF function in depression and anxiety. Nat Neurosci 10(9): 1089-1093.

51. Chen Biao, Dowlatshahi Dar, MacQueen Glenda M, Wang Jun-Feng, Young L Trevor (2001) Increased Hippocampal BDNF Immunoreactivity in Subjects Treated with Antidepressant Medication. World J Biol Physcia 50(4): 260-265.

52. Lazar MA, McIntyre RS (2019) Novel Therapeutic Targets for Major Depressive Disorder. Neurobiol Dep 2019: 383-400. 